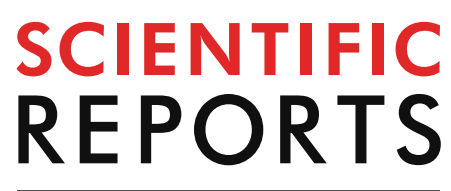

natureresearch

Check for updates

\title{
Patterns of DNA variation between the autosomes, the $X$ chromosome and the $Y$ chromosome in Bos taurus genome
}

\begin{abstract}
Bartosz Czech $^{1 \bowtie}$, Bernt Guldbrandtsen ${ }^{2,3}$ \& Joanna Szyda ${ }^{1,4}$
The new ARS-UCD1.2 assembly of the bovine genome has considerable improvements over the previous assembly and thus more accurate identification of patterns of genetic variation can be achieved with it. We explored differences in genetic variation between autosomes, the $X$ chromosome, and the $Y$ chromosome. In particular, variant densities, annotations, lengths (only for InDels), nucleotide divergence, and Tajima's D statistics between chromosomes were considered. Wholegenome DNA sequences of 217 individuals representing different cattle breeds were examined. The analysis included the alignment to the new reference genome and variant identification. 23,655,295 SNPs and 3,758,781 InDels were detected. In contrast to autosomes, both sex chromosomes had negative values of Tajima's $D$ and lower nucleotide divergence. That implies a correlation between nucleotide diversity and recombination rate, which is obviously reduced for sex chromosomes. Moreover, the accumulation of nonsynonymous mutations on the $\mathrm{Y}$ chromosome could be associated with loss of recombination. Also, the relatively lower effective population size for sex chromosomes leads to a lower expected density of variants.
\end{abstract}

DNA variation refers to differences in DNA sequence among individuals. Decreasing costs and reducing the time of whole-genome sequencing using the Next-Generation Sequencing (NGS) technology brings the opportunity to sequence many samples. The analysis of differences in DNA variation between autosomes and sex chromosomes plays an important role in understanding the evolution of chromosomes. In terms of studying variation, cattle is an interesting model, since for several generations it has undergone strong artificial selection toward increased milk (Holstein, Jersey, Jysk, Rd Dansk Malkerace anno 1970, Sortbroget Dansk Malkerace anno 1965) or beef (Danish Shorthorn) production. Besides, modern cattle is composed of breeds with distinct phenotypic characteristics. We used the new ARS-UCD 1.0.25 assembly that is the latest and the most accurate (less gaps) version of the cattle reference genome ${ }^{1}$. However, this assembly, like the UMD3.12, does not contain the bovine Y chromosome (BTY), so most analyzes ignored the Y chromosome. As a result it has been omitted from most studies, so that in livestock, only one association was identified between genetic variants from BTY and phenotypes (in particular in pigs, source AnimalQTdb www.animalgenome.org/cgi-bin/QTLdb/index; Release 41). Recently the 1000 Bull Genomes Project ${ }^{3}$ added the Y chromosome sequence from the Btau 5.0.1 to the ARS-UCD, creating the ARS-UCD1.2_Btau5.0.1Y assembly. Thereby has become feasible to use all nuclear bovine chromosomes.

According to the ARS-UCD1.2_Btau5.0.1Y reference genome assembly, the bovine genome spans 2,759,153,975 bps with 30,278 genes (GeneBank assembly accession: GCA_002263795.2 and GCA_000003205.6) and consists of 29 autosomes and two sex chromosomes-X and Y (Table 1). The hemizygous Y chromosome in Bos taurus is short and contains only a few genes. In contrast, the bovine X chromosome (BTX) contains many more genes and its length is similar to the BTA2. Chromosome 1 is the longest bovine chromosome, spanning $158,534,110$ bps with 1,218 genes, while chromosome 25 is the shortest bovine chromosome $(949,746$ bps shorter than BTY), spanning 42,350,435 bps with 1,611 genes. Regarding the ratio of the number of genes to the total

\footnotetext{
${ }^{1}$ Biostatistics Group, Department of Genetics, Wroclaw University of Environmental and Life Sciences, Kozuchowska 7, 51-631 Wrocław, Poland. ${ }^{2}$ Center for Quantitative Genetics and Genomics, Department of Molecular Biology and Genetics, Aarhus University, 8830 Tjele, Denmark. ${ }^{3}$ Department of Animal Sciences, University of Bonn, Endenicher Allee 15, 53115 Bonn, Germany. ${ }^{4}$ Institute of Animal Breeding, Krakowska 1, 32-083 Balice, Poland. ${ }^{\circledR}$ email: bartosz.czech@upwr.edu.pl
} 


\begin{tabular}{|c|c|c|c|}
\hline Chromosome & Length of chromosome [bp] & Number of genes & Number of genes/Mbp ratio \\
\hline 1 & $158,534,110$ & 1216 & 7.67 \\
\hline 2 & $136,231,102$ & 1284 & 9.43 \\
\hline 3 & $121,005,158$ & 1287 & 10.64 \\
\hline 4 & $120,000,601$ & 593 & 4.94 \\
\hline 5 & $120,089,316$ & 1080 & 8.99 \\
\hline 6 & $117,806,340$ & 692 & 5.87 \\
\hline 7 & $110,682,743$ & 1264 & 11.42 \\
\hline 8 & $113,319,770$ & 898 & 7.92 \\
\hline 9 & $105,454,467$ & 800 & 7.59 \\
\hline 10 & $103,308,737$ & 1554 & 15.04 \\
\hline 11 & $106,982,474$ & 1620 & 15.14 \\
\hline 12 & $87,216,183$ & 1194 & 13.69 \\
\hline 13 & $83,472,345$ & 479 & 5.74 \\
\hline 14 & $82,403,003$ & 864 & 10.49 \\
\hline 15 & $85,007,780$ & 738 & 8.68 \\
\hline 16 & $81,013,979$ & 1127 & 13.91 \\
\hline 17 & $73,167,244$ & 446 & 6.10 \\
\hline 18 & $65,820,629$ & 948 & 14.40 \\
\hline 19 & $63,449,741$ & 526 & 8.29 \\
\hline 20 & $71,974,595$ & 387 & 5.38 \\
\hline 21 & $69,862,954$ & 412 & 5.90 \\
\hline 22 & $60,773,035$ & 845 & 13.90 \\
\hline 23 & $52,498,615$ & 1708 & 32.53 \\
\hline 24 & $62,317,253$ & 1048 & 16.82 \\
\hline 25 & $42,350,435$ & 1611 & 38.04 \\
\hline 26 & $51,992,305$ & 849 & 16.33 \\
\hline 27 & $45,612,108$ & 1665 & 36.50 \\
\hline 28 & $45,940,150$ & 982 & 21.38 \\
\hline 29 & $51,098,607$ & 733 & 14.34 \\
\hline $\mathrm{X}$ & $139,009,144$ & 1222 & 8.79 \\
\hline $\mathrm{Y}$ & $43,300,181$ & 206 & 4.76 \\
\hline
\end{tabular}

Table 1. Summary of ARS-UCD1.2_Btau5.0.1Y reference genome.

contig length (chromosome), we can see that BTA25 has the highest ratio (38.04), while the Y chromosome has the lowest ratio (4.76). It is also worth to mention, that the karyogram of cattle chromosomes reports BTA29 as the shortest chromosome. BTY contains the lowest number of genes (206), while chromosome 23 contains the highest number of genes $(1,708)$. Clearly, chromosome length is not linearly related to the number of genes. Moreover, 29 autosomes are acrocentric, while both sex chromosomes (X and Y) are submetacentric. Since recombination that generates new combinations of alleles is characteristic to autosomes, on sex chromosomes, this phenomenon is reduced in males to only small homologous regions shared between BTX and -Y, called the pseudoautosomal regions (PARs). BTY is poorly characterized because it is difficult to sequence due to the occurrence of a high proportion of repetitive sequences ${ }^{4}$.

Differences in genetic variation patterns arise from variable recombination and mutation rates, genetic drift, demography, selection, and population history. Therefore the focus of our study was on the comparison of patterns of genetic variation between autosomes, the $\mathrm{X}$ chromosome, and the $\mathrm{Y}$ chromosome in the context of the bovine genome.

\section{Results}

Alignment to the reference genome. The quality of alignment was expressed by the total percent of mapped reads and the percent of properly paired mapped reads (both reads are mapped close to each other in opposite directions on the same chromosome). The percent of mapped reads for each individual was very high and ranged from $91.4 \%$ to $99.9 \%$ with mean $99.7 \%( \pm 0.6)$ and very similar mode $99.9 \%$. The percent of properly paired mapped reads was also high and varied between $89.3 \%$ and $99.1 \%$ with mean $97.1 \%( \pm 1.7)$ and mode 97.9\%. Average genome coverage was calculated separately for each individual and ranged from 5.28 to 46.79 with mean and mode both equal to 25 (Fig. 1). Individuals average genome coverage less than 15, while two individuals have the average genome coverage above 40 . The individual with the lowest average genome coverage (5.28) contains $99.6 \%$ of mapped reads and $97.8 \%$ of properly paired mapped reads. The individual with the highest average coverage (46.79) contain $99.9 \%$ of mapped reads and $97.9 \%$ of properly paired mapped reads. 


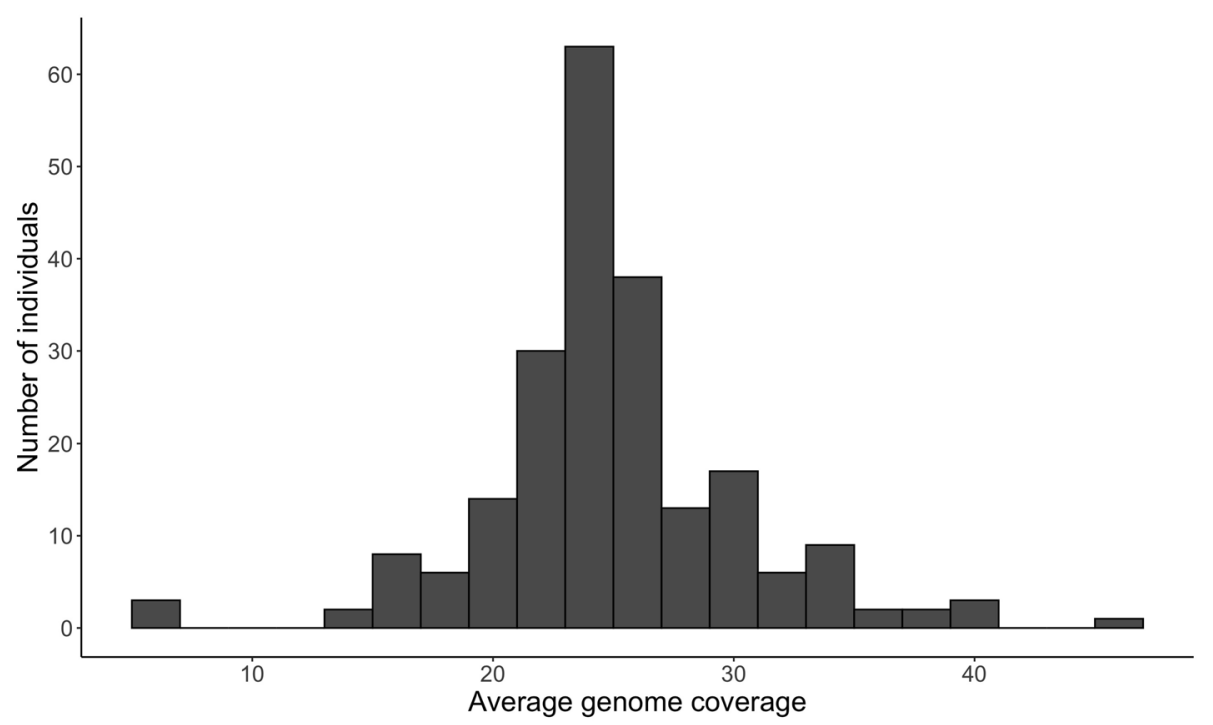

Figure 1. Average genome coverage.

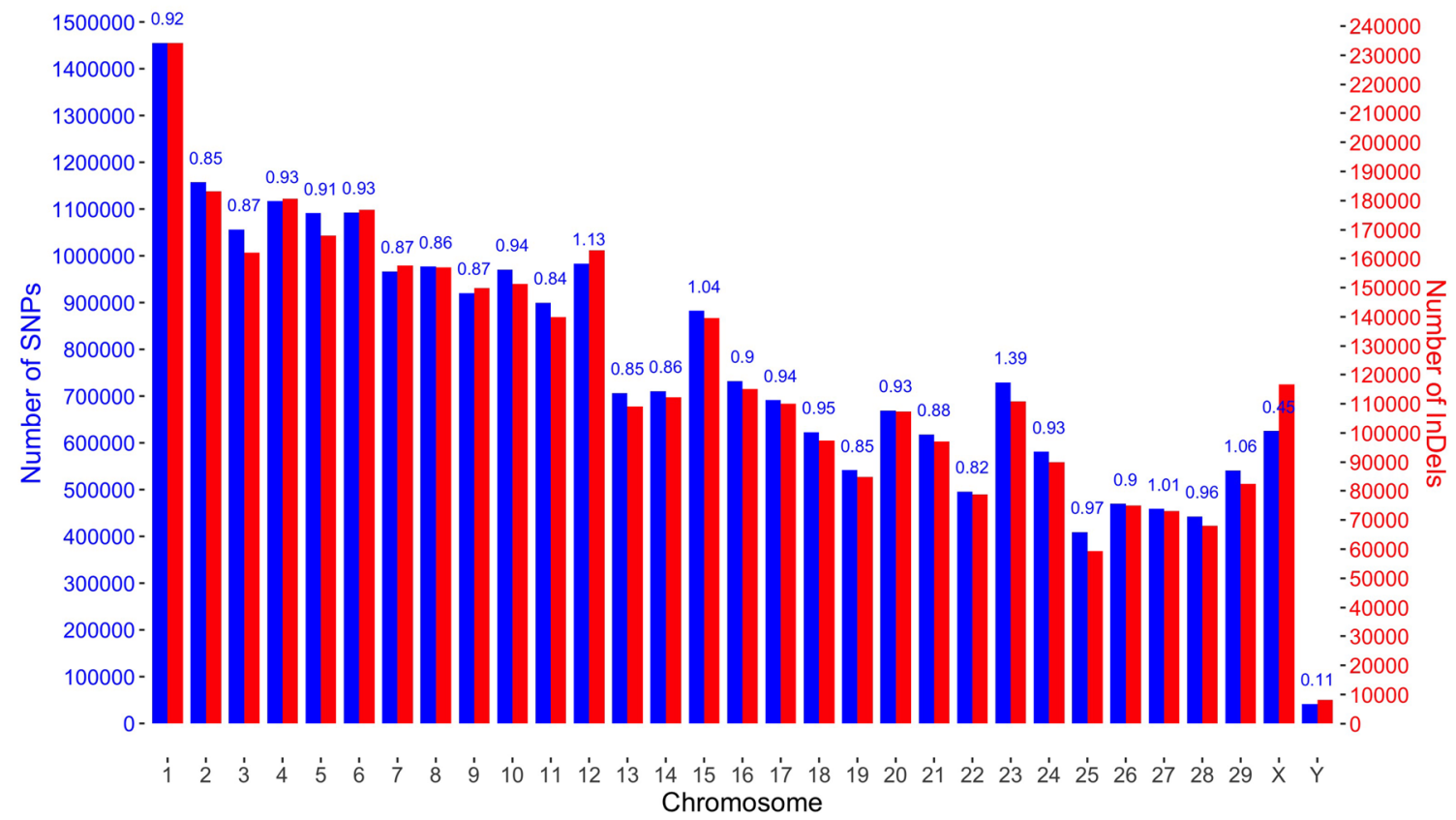

Figure 2. The distribution of variants. Blue bars with the y-axis on the left side represent SNPs, while red bars with the $y$-axis on the right side represent InDels. Values above bars represent the percent of the total length of chromosome covered by SNPs.

Variation. Overall, 27,414,076 variants were identified (Fig. 2). Of these, $86.3 \%$ were SNPs, and $13.7 \%$ were InDels. BTA1 contained the highest number of variants $(1,689,556)$, while the BTY contained the lowest number of variants $(49,591)$. The total number of SNPs was $23,655,295,0.9 \%$ of the total genome length. $9,848,025$ SNPs were located within coding sequences (CDS). BTA1 contained the highest number of SNPs $(1,455,295$ with 530,272 SNPs in coding regions), but the highest SNP density, expressed by the proportion of the number of SNPs to chromosome length, was highest for BTA25 (2.6\%). BTY was characterized by the lowest number of SNPs $(41,500 ; 4,185$ in coding regions) and the lowest SNP density (0.1\%). 3,758,781 of InDels were identified including 1,591,937 in coding regions. BTA1 had the highest number of InDels $(234,261)$, whereas BTY had the lowest number of InDels (8,091) (Fig. 2).

The length of InDels varied between 1 and 281 bps on autosomes, from 1 to 233 bps on BTAX, and from 1 to $156 \mathrm{bps}$ on BTY and is not uniformly distributed $(P<0.001)$. The most frequently observed length of InDels was one bp, but the median length was two bps (Fig. 3). InDel median length differed significantly $(P<0.001)$ 


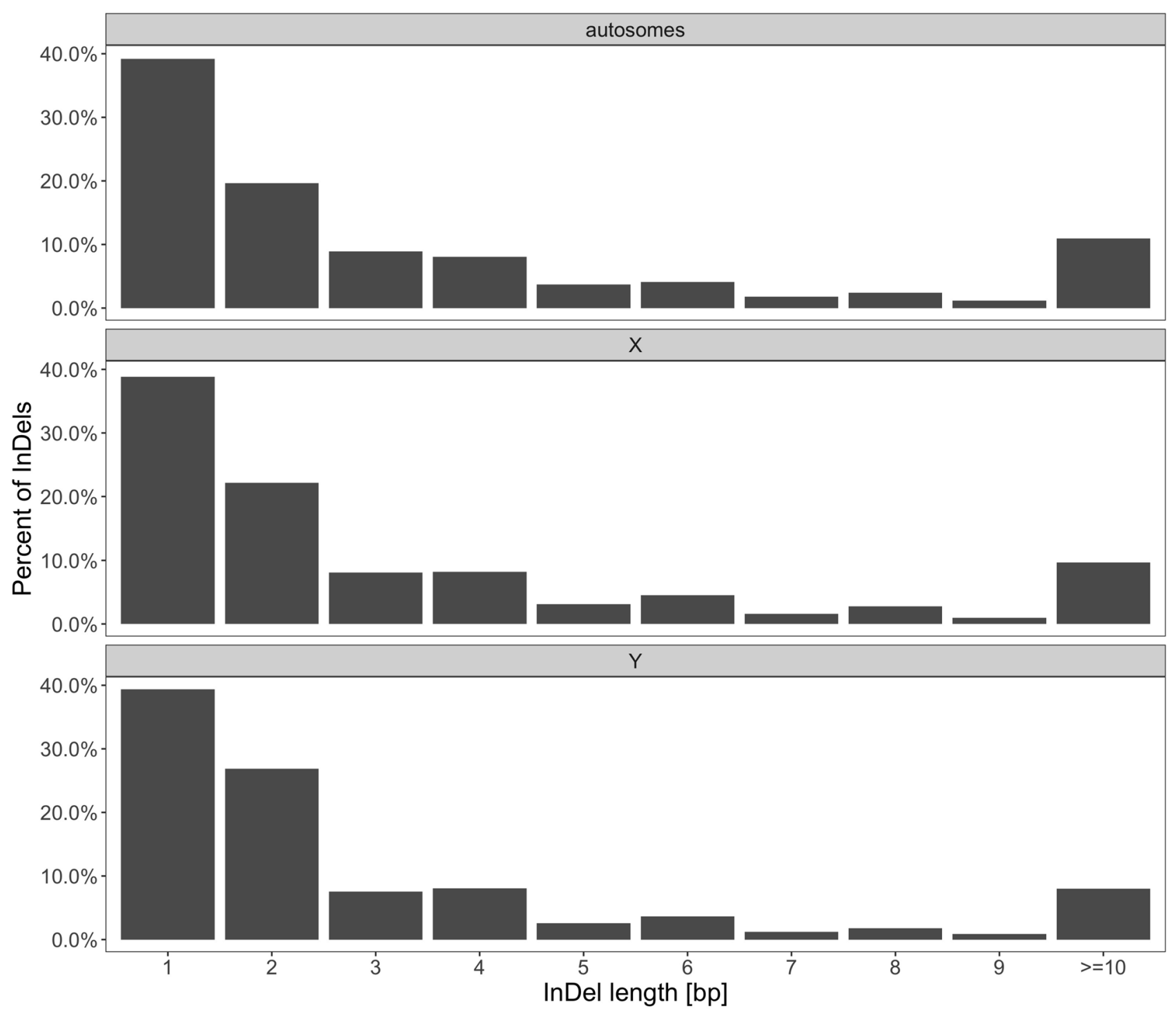

Figure 3. Length distribution of deletions and insertions for autosomes and sex chromosomes.

between autosomes and BTX as well as between autosomes and BTY. 12\%, 19\%, and 98\% of indels located respectively on autosomes, BTX and, BTY, was longer or equal 10bps.

Distribution of polymorphisms. The analysis of variant distribution was carried out by counting the number of variants within $100 \mathrm{kbp}$ non-overlapping windows (Fig. 4). The number of SNPs per $100 \mathrm{kbp}$ bin was heterogeneous across windows within the same chromosome $(P<0.001)$. The highest number of SNPs in one window $(16,184)$ was observed on BTA12. On the other hand, on four chromosomes we identified windows without SNPs: BTA8 (one window), BTA9 (eight windows), BTA10 (eight windows), and BTY (58 windows). All of the windows were annotated as intergenic. However, 27 out of the total 58 windows on BTY corresponded to gaps in the reference genome marked by stretches of Ns. Conversely, windows with the number of SNPs exceeding 10,000 were found on BTA4 (one window with 10,838 SNPs) $8 \%$ of window length overlapped with genes, BTA12 (four windows with 10,431, 11,487, 15,097 and 16,186 SNPs) in 3\% of windows length overlapped with genes, and BTA23 (three windows with 11,706; 12,452 and 13,229 SNPs) in which 100\% of windows length overlapped transcription regions. Interestingly, the functional annotation showed that four out of the eight SNP-rich windows overlapped with lncRNA genes.

The highest overall number of InDels was identified on BTA23 (2,930 InDels). InDel density (Fig. 5) was not uniform across the genome $(P<0.001)$. We identified 122 windows without InDels-seven on BTA9, eight on BTA10, and 107 on BTY. All the windows on BTA7 and BTA8 were intergenic. On BTY, 64 of the windows were intergenic, whereas 35 windows corresponded to gaps in the reference assembly. However, eight windows intersected 10 genes. $1 \%$ to $13 \%$ of these windows overlapped with the sequence of a gene. Windows harboring more than 2,000 InDels were found on BTA4 (one window with 2,162 InDels), BTA12 (two windows with 2,405 and 2,792 InDels), and BTA23 (three windows with 2,022; 2,258, and 2,930 InDels). Each of these windows 


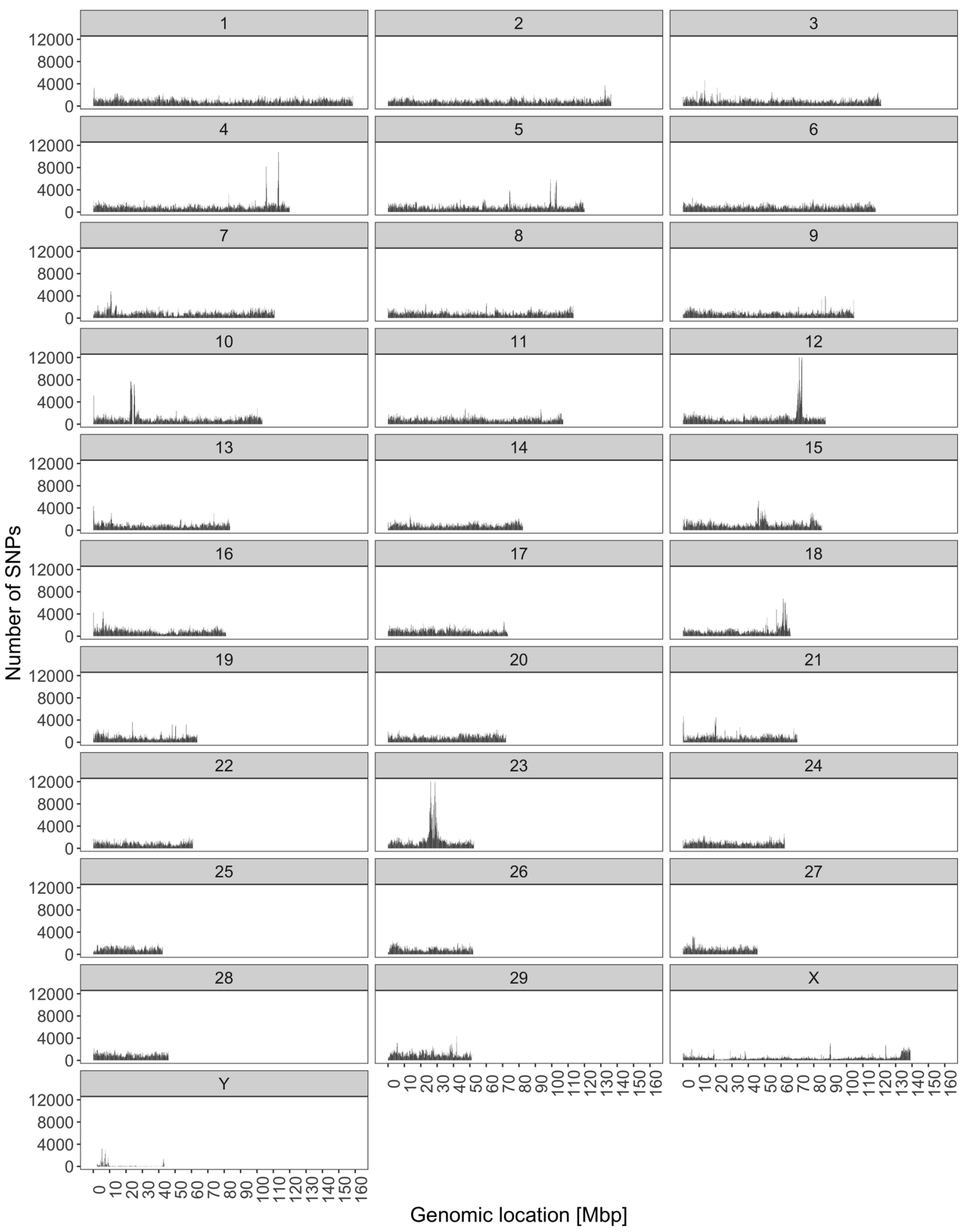

Figure 4. The distribution of SNPs across chromosomes.

overlapped with genes comprising from $33 \%$ of a window length to $100 \%$ of length, both on BTA23. As for SNPrich windows, three out of the six InDel-rich windows overlapped with lncRNA genes.

Variant annotation. The annotation of variants expressed as the percent of polymorphic bases located in non-coding and coding regions in relation to their length was summarized in Fig. 6. For each chromosome, the proportion of number of polymorphic sites to the length of the specific region (coding or non-coding) was higher for coding regions in comparison to non-coding regions. The proportion of the number of variants 


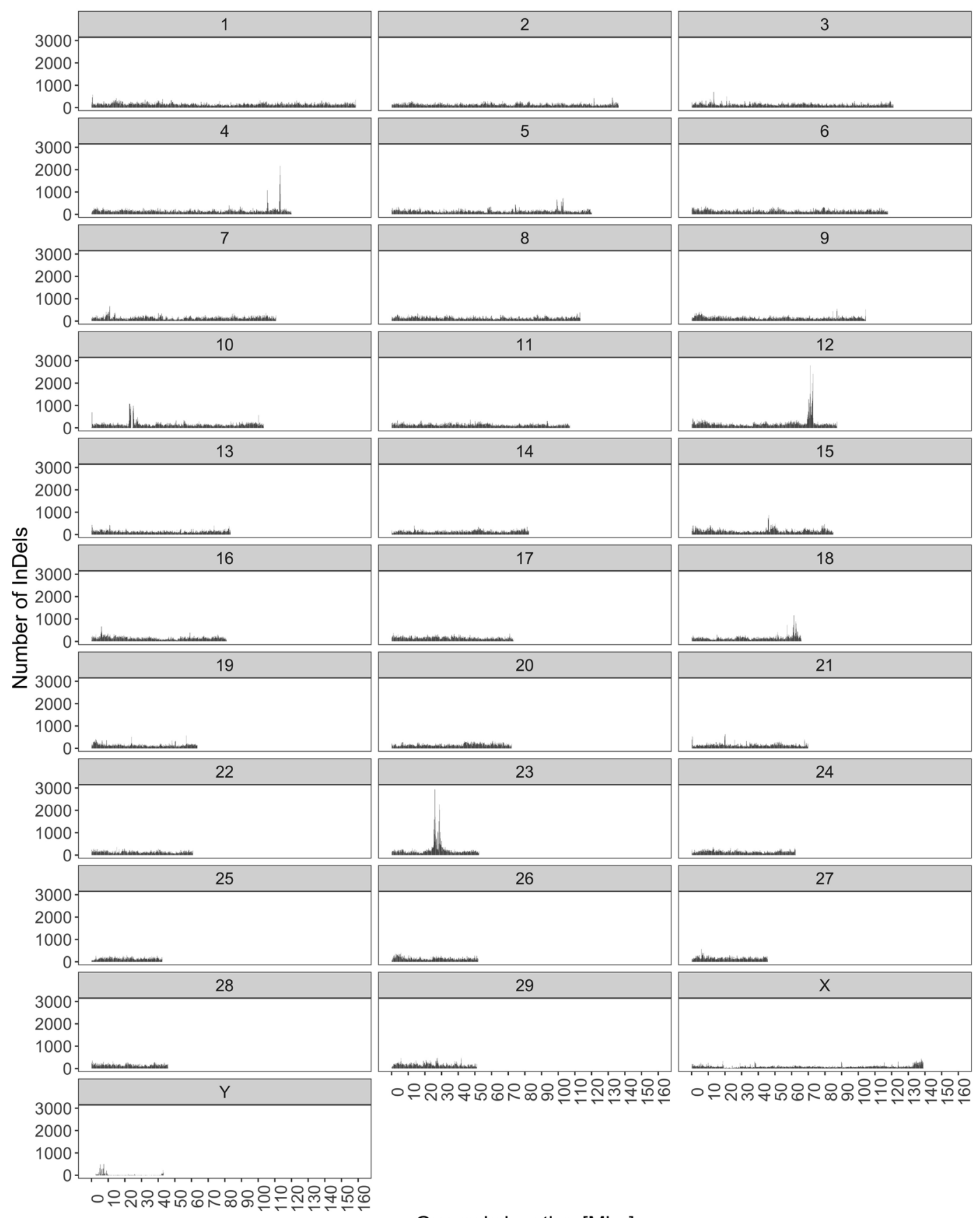

\section{Genomic location [Mbp]}

Figure 5. The distribution of InDels across chromosomes.

overlapping coding regions to the total length of this region was the highest on autosomes $(9.90 \pm 3.20$ ), while the lowest on BTY (1.83). The proportion of the number of variants overlapping non-coding regions to the total length of this region was also observed on autosomes $(0.65 \pm 0.09)$ with the lowest proportion on BTY $(0.10)$.

Focusing only on genes, on autosomes and BTX we observed the highest percent of variants was located in introns $(41.8 \% \pm 2.9 \%$ and $42.6 \%$, respectively) and in non-coding transcripts $(41.7 \% \pm 2.4 \%$ and $41.7 \%$, respectively). The $\mathrm{Y}$ chromosome showed the highest percent of variants in regions annotated up to 5,000 bps upstream of the $3^{\prime}$ end of gene start (25.3\%) and up to 5,000 bps downstream of the $5^{\prime}$ end of gene end (24.8\%). It was accompanied by a proportion of intronic and non-coding transcript variants being by $20 \%$ lower than in BTA and BTX. Moreover, variants with high (splice acceptor, splice donor, stop gained, frameshift variant, stop lost, start lost) and moderate (missense) impacts on proteins occurred more frequently on BTY than on BTA and BTX, while the latter had the lowest percent of variants with high or moderate impacts. Interestingly, on BTY $0.11 \%$ of variants in transcription regions were frameshift, which is much higher than on BTA $(0.02 \%)$ and 

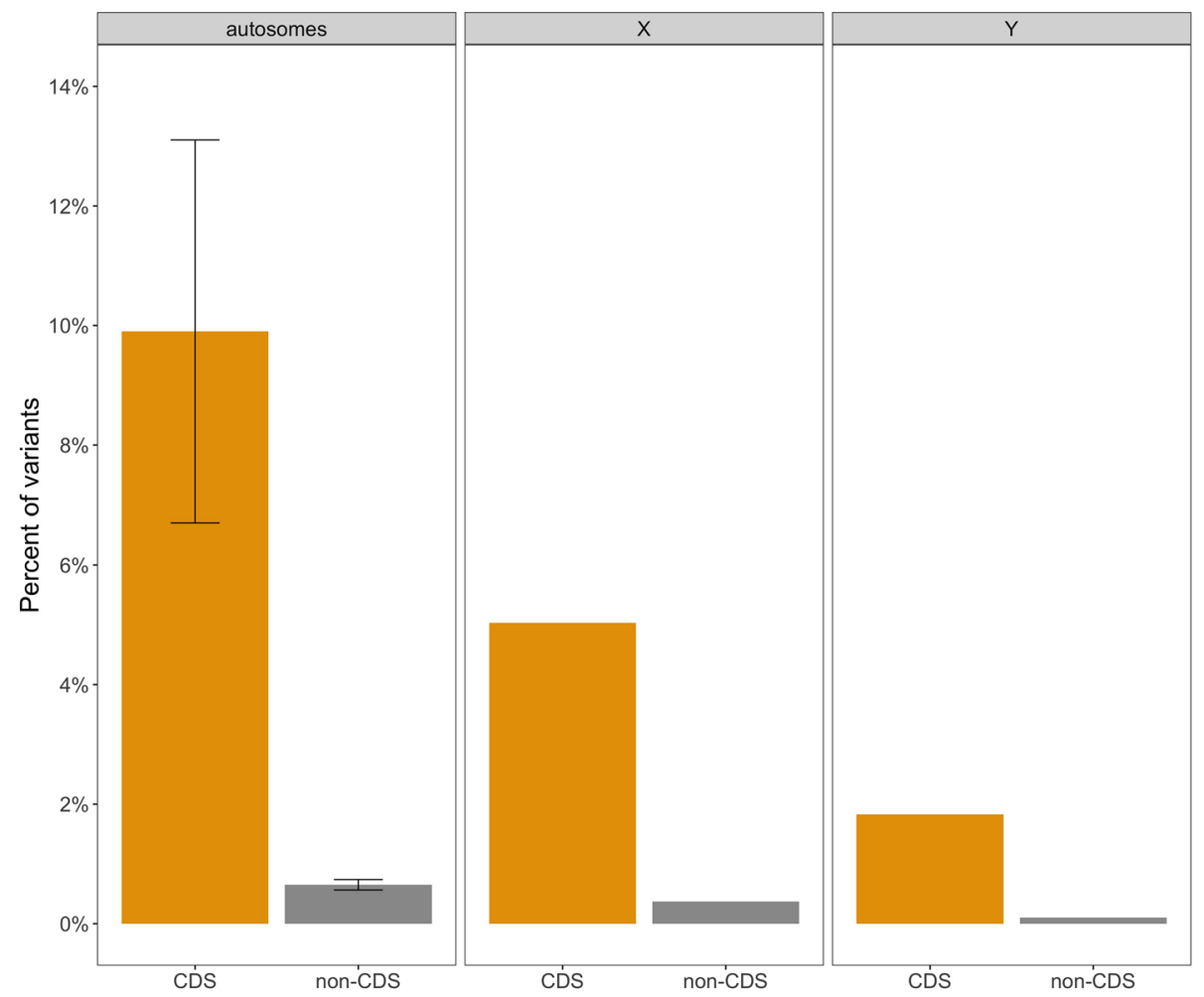

Figure 6. The annotation of variants in coding (CDS) and non-coding (non-CDS) regions.

BTX (0.01\%). The same tendency was observed for stop gained variants, which constituted $0.03 \%$ of BTY, but only $0.004 \%$ of BTA and $0.003 \%$ of BTX.

Nonsynonymous and missense variants were examined in order to predict whether an amino acid substitution affects protein function. For this purpose, the SIFT score was predicted. A variant with score 0 to 0.05 is considered deleterious while $>0.05$ are considered tolerated. Figure 7 reports the distribution of SIFT scores among nonsynonymous and missense variants. We can see, that the distributions of SIFT scores for autosomes and the $\mathrm{X}$ chromosome are the same $(P=0.76)$, while differences between the $\mathrm{X}$ chromosome and the $\mathrm{Y}$ chromosomes as well as between the autosomes and the $\mathrm{Y}$ chromosomes are different $(P<0.001)$. The $\mathrm{Y}$ chromosome has the highest median of the SIFT score.

Population genetics. The ratio of nonsynonymous to synonymous SNPs $\left(K_{a} / K_{s}\right.$ ratio) was the highest for BTY (2.00) and the lowest for BTX (0.62). Autosome averaged ratio was also below unity $(0.79 \pm 0.15)$ and varied between 0.57 for BTA22 and 1.10 for BTA4, BTA15, BTA18, and BTA23. Tajima's D statistic significantly differed between all three chromosome groups $(P<0.001)$ and was positive for autosomes and negative for both sex chromosomes (Fig. 8). Nucleotide diversity had the highest median for autosomes, followed by BTX and BTY (Fig. 9). All differences in distributions of nucleotide divergence between chromosome groups were significant $(P<0.001)$. Furthermore, windows with extremely high diversity and windows with extreme values of Tajima's $D$ statistic were examined for their SNP density. These windows do not overlap regions with high SNP density.

\section{Discussion}

Our goal with this study was to compare patterns of genetic variation between autosomes, the BTX, and the BTY. A high percent of mapped reads, a high percent of properly mapped paired reads, and the fact that the most of individual genomes were sequenced with a genome average coverage exceeding twenty, demonstrated high quality of our data allowing for reliable inferences. Interestingly, individuals with low percent of mapped reads and low percent of properly mapped reads had high genome average coverage. This implies no correlation between genome average coverage and percent of mapped and properly mapped reads.

Altogether, over 23.6 million SNPs and over 3.7 million InDels were identified, among them $24 \%$ of SNPs and $94 \%$ of InDels were novel. Such proportion between novel SNPs and InDels can be caused by the fact that many more studies have been related to SNPs and thus the SNP database is more complete ${ }^{5}$. A larger number of SNPs than InDels is not an uncommon observation. InDels in transcription regions are highly deleterious to gene function as they can completely alter protein amino acid sequence by changing the open reading frame (frameshift mutation). BTY contains the lowest number of variants, which is not only due to its length. For instance, BTA25 is the shortest autosome, but it contains much higher number of variants. Taking into account the length of each chromosome, we observed that only $0.1 \%$ of the whole length of the BTY contained SNPs, where it is $0.45 \%$ for 


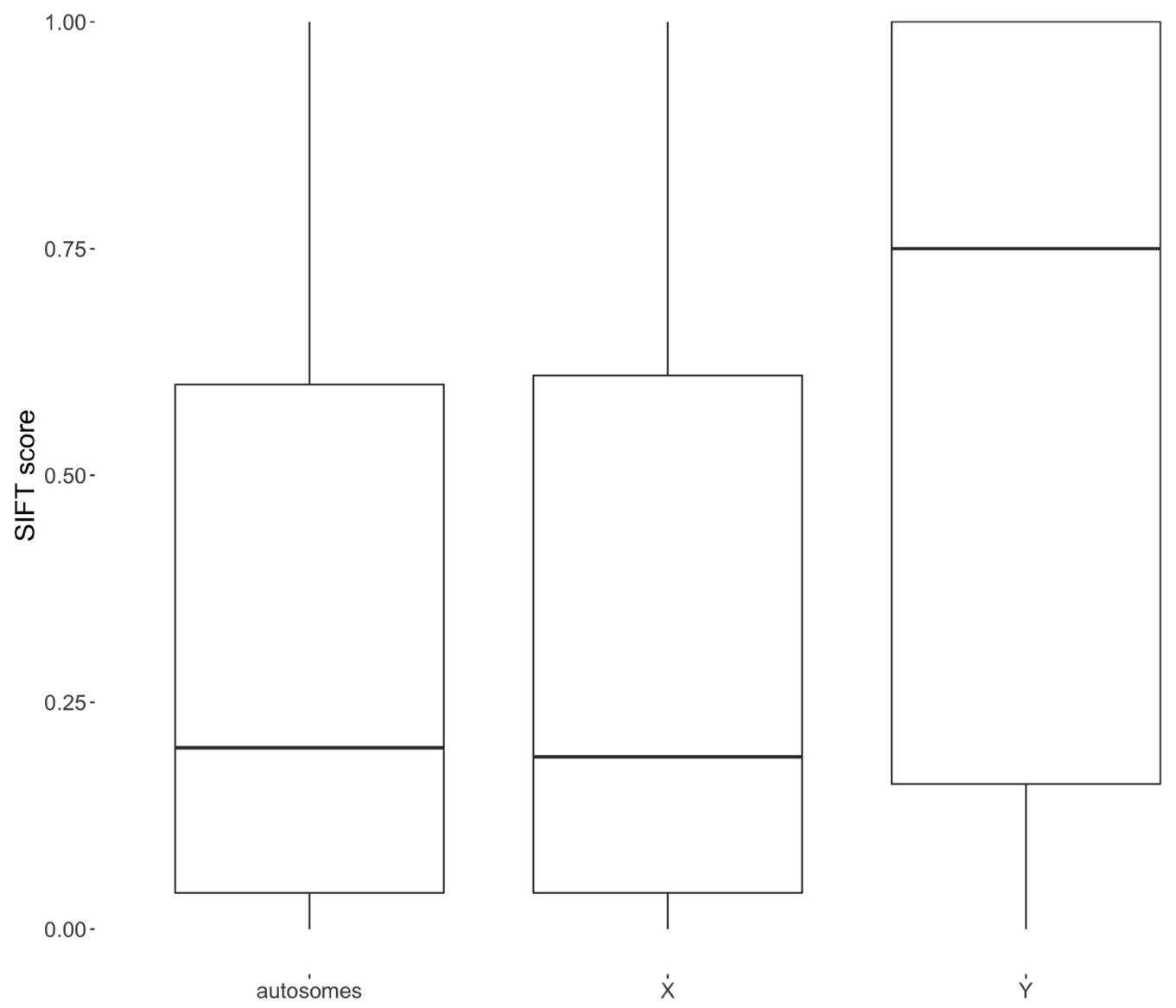

Figure 7. SIFT score for nonsynonymous and missense variants on autosomes, the BTX, and the BTY.

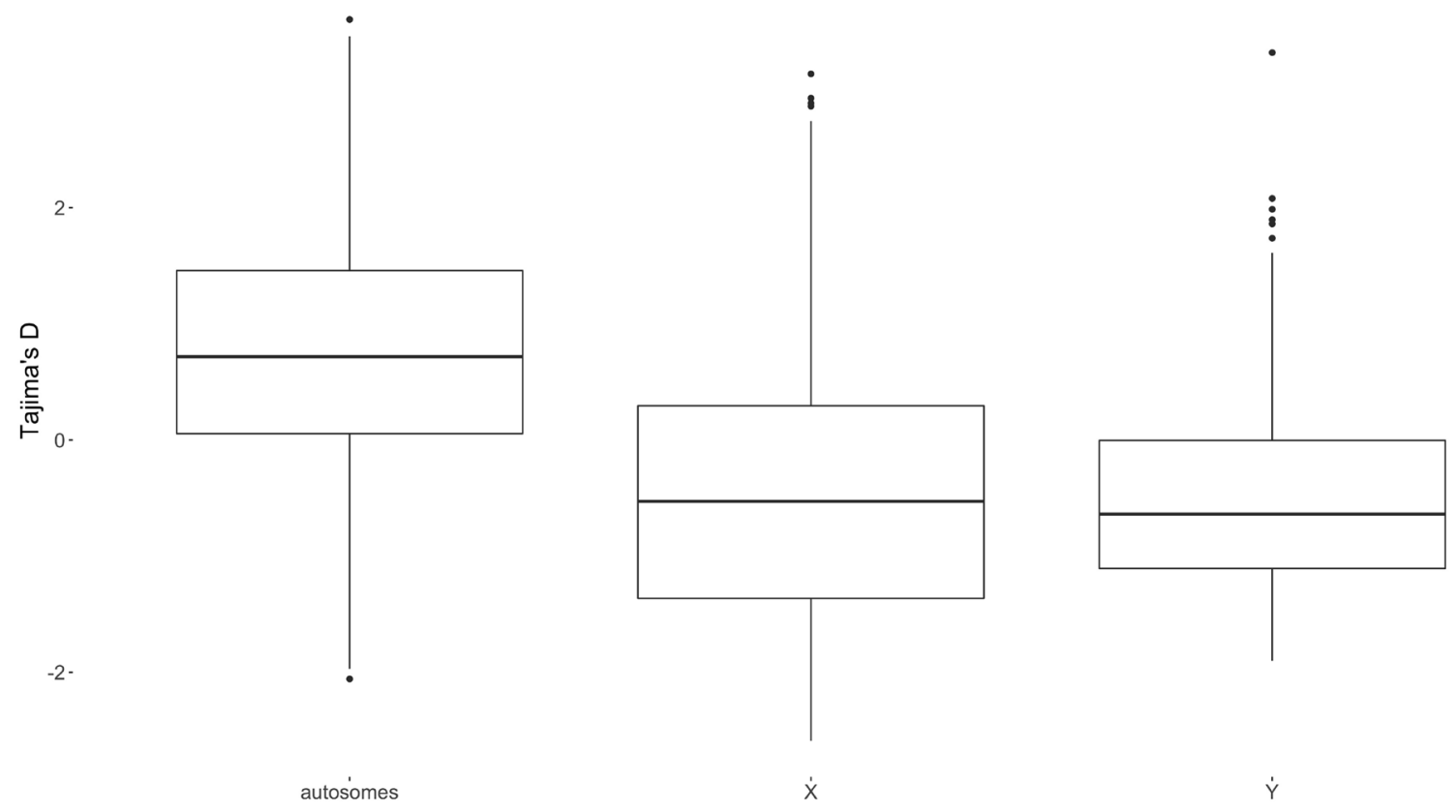

Figure 8. Tajima's $D$ for autosomes, the BTX chromosome, and the BTY chromosome. 


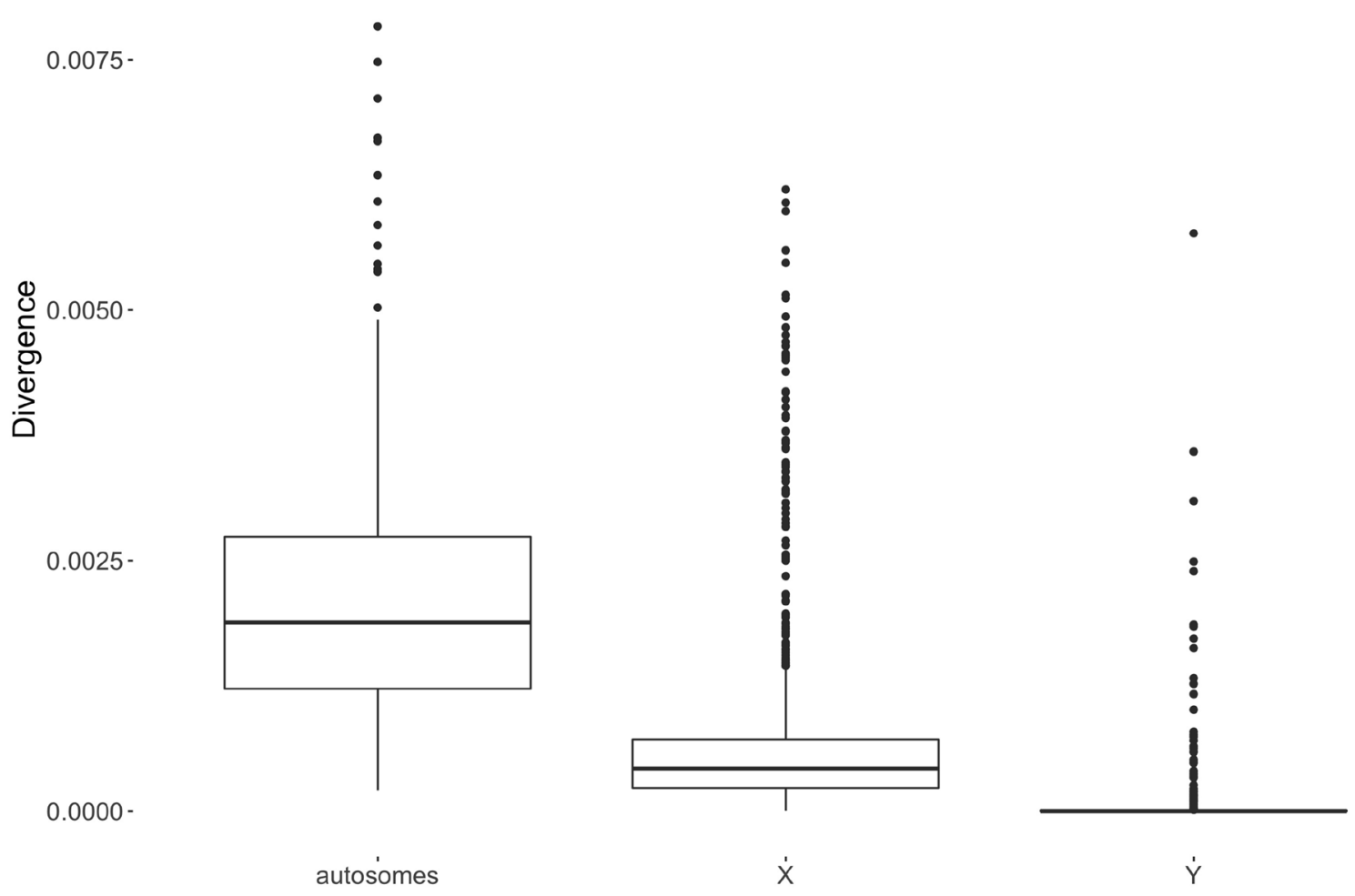

Figure 9. Nucleotide divergence for autosomes, the BTX chromosome, and the BTY chromosome.

BTX. On the other hand, the shortest chromosome (BTA25) exhibited the highest SNP density of 2.58\%. One of the factors affecting DNA variation is the effective population size $\left(N_{e}\right)$. Estimated based on autosomes, the effective population size is higher than based on sex chromosomes-3/4 of autosomal $N_{e}$ for X and $1 / 4$ of autosomal $N_{e}$ for $\mathrm{Y}^{6}$. The lower $N_{e}$, the more important role of genetic drift, so we expect that the effect of genetic drift is the highest for the $\mathrm{Y}$ chromosome, which furthermore implies lower neutral diversity of $\mathrm{Y}^{7}$. This observation contradicts the theory of male mutation bias that assumes that more mutations accumulate in male germline due to a greater number of male germline cell divisions, which is especially pronounced for BTY which "spends more time" in male germline than the other chromosomes ${ }^{8}$. A difference observed in our study is possibly due to gaps in the BTY chromosome assembly of the bovine genome (i.e. a high number of unknown nucleotides), which humper an accurate comparison of variant density between autosomes, BTX and BTY.

However, more recent studies emphasize that the comparison of mutation patterns on a general, chromosomewide scale, is not valid due to a strong variation in local mutation rates ${ }^{9,10}$. Also in our study SNP and InDel distribution showed a non-random pattern, characterized by mutation hotspots with very high variant density, albeit only on autosomes. We observed that SNP density is positively correlated with indel density, which is in agreement with results obtained for humans by Hodgkinson et al. ${ }^{11}$. Estivill et al. ${ }^{12}$ showed that regions with highdensity of SNPs are correlated with segmental duplications in the human genome. Varela and Amos ${ }^{13}$ declared that regions with unusually high recombination rates tend to have a high density of SNPs, while Aggarwala and Voight ${ }^{14}$ estimated the effect of DNA sequence k-mers on SNP probability.

Based on the annotation of transcription regions, it can be shown that there is a tendency that variants in transcription regions on BTX have less severe consequences as compared to Y and autosomes. Fewer extreme variants are consistent with purging due to the hemizygous state in males. On autosomes, these would most of the time be hidden in males due to diploidy. Based on $\mathrm{Ka} / \mathrm{Ks}$ ratio, we also observed that on the BTY there is a tendency to accumulate more nonsynonymous than synonymous substitutions $(\mathrm{Ka} / \mathrm{Ks}=2.00)$. In contrast, BTX shows a much lower ratio $(\mathrm{Ka} / \mathrm{Ks}=0.62)$. Such differences might arise from the tendency for the degeneration of the Y chromosome-Haldane's rule, which indicates faster male evolution and reason of accumulation of nonsynonymous variants, mainly due to the lack of the recombination ${ }^{15}$. In males, BTX is hemizygous, so any, even slightly deleterious mutation has an effect on the phenotype. For mammalian genomes such situation was demonstrated in a simulation study by Mackiewicz et al. ${ }^{16}$

\section{Methods}

Material. The material comprised whole-genome DNA sequences of 217 individuals representing Holstein (69 bulls and 6 cows), Jersey (41 bulls), Jysk ( 5 bulls), Rd Dansk Malkerace (46 bulls), Rd Dansk Malkerace anno 1970 (15 bulls and 5 cows), Sortbroget Dansk Malkerace anno 1965 (15 bulls and 5 cows) and Danish 
Shorthorn ( 5 bulls and 5 cows) breeds available courtesy of the Center for Quantitative Genetics and Genomics at the Aarhus University. Animals were not closely related. There can be found some distant relationship within the breeds.

Whole-genome DNA sequences were obtained by the Illumina HiSeq 2000 Next Generation Sequencing platform and similar short-read sequencing platforms. Access to this data was available via the computer cluster of the Center for Quantitative Genetics and Genomics at Aarhus University. ARS-UCD1.2_Btau5.0.1Y ${ }^{1}$ reference genome was used to processing whole-genome sequence data. This genome represents the latest reference genome of Bos taurus additionally assembled with the $\mathrm{Y}$ chromosome (Btau5.0.1) from Baylor College ${ }^{17}$. In this study two GFF (General Feature Format) files were used for the annotation of identified variants. Btau_5.0.1 and ARS-UCD1.2 GFF were merged to obtain a complete annotation file for each chromosome. Those files were downloaded from the NCBI database (ftp://ftp.ncbi.nlm.nih.gov/genomes/all/ GCF/002/263/795/GCF_002263795.1_ARS-UCD1.2/ and ftp://ftp.ncbi.nlm.nih.gov/ genomes/all/GCF/000/003/205/GCF 000003205.7 Btau_5.0.1/). The actual assembly file used was downloaded from https://sites.ualberta.ca/ stothard/1000_bull_genomes/ARS-UCD1.2_Btau5.0.1Y. fa.gz.

Processing the next-generation sequencing data. The FastQC software was used to summarize and visualize sequence quality ${ }^{18}$. Sequence quality metrics measure the probability that a given base is called incorrectly. Low quality bases were trimmed from reads using Trimmomatic ${ }^{19}$ with options SLIDINGWINDOW:3:15, LEADING:3, TRAILING:3, and a minimum read length of $70 \mathrm{bp}$. Cleaned reads were aligned to the reference genome by using the Burrows-Wheeler Aligner (BWA) with the MEM algorithm ${ }^{20}$. After alignment, the flagstat tool in SAMtools ${ }^{21}$ was used to calculate the percent reads correctly mapped to the reference genome. The genome average coverage, representing the number of times that a base in the reference genome was covered by aligned reads ${ }^{22}$, was calculated for each individual, using the genomecov tool in bedtools ${ }^{23}$. It was expressed by coverage $=\frac{R \cdot L}{G}$, where $R$ is the total number of aligned reads, $L$ is average read length and $G$ is the genome size. The output from BWA-MEM was piped to SAMtools ${ }^{21}$ and then compressed to the BAM format (Binary Alignment Map) - a binary version of the SAM format. Afterwards, SAMtools fixmate ${ }^{21}$ was run to adjust the materead position. BAM files were sorted using SAMtools ${ }^{21}$. PCR duplicates were marked using MarkDuplicates from Picard ${ }^{24}$. Finally, Base quality scores were recalibrated using Genome Analysis Toolkit (GATK) ${ }^{25}$.

Variant calling. Variant calling allows the identification of differences between analyzed sequences and the reference genome, i.e., of polymorphic sites. First, GATK's HaplotypeCaller ${ }^{25}$ was used to create files (gVCF files) that summarize information on sites potentially deviating from the reference. Specifically, the tool identifies genomic regions, so-called ActiveRegions, which contain significant differences from the reference genome. Those regions are then processed by HaplotypeCaller. Other variations (non ActiveRegions) are skipped in order to accelerate the analysis. Afterward, ActiveRegions are used to construct haplotypes by building a De Bruijnlike graph ${ }^{26}$ and calculate haplotype frequencies. Haplotypes were realigned against the reference haplotype using the Smith-Waterman algorithm ${ }^{27}$ to identify potentially polymorphic sites. Then a matrix of likelihoods of haplotypes given the DNA sequence of reads was calculated using Hidden Markov Models. Thereafter, HaplotypeCaller assigned the most likely genotypes ${ }^{25}$. The standard phred-scaled confidence threshold of 30 was used to remove the potential false-positive variants. The last step of variant calling comprised merging gVCF files representing different individuals. For this purpose, GATK GenotypeGVCFs was used ${ }^{25}$. This step resulted in raw variant call files (VCF), which contained summary information of each detected variant.

After variant calling, the identified polymorphisms were annotated with predicted biological consequences and functions. For each polymorphic variant the associated Sequence Ontology $(\mathrm{SO})^{28}$ terms, which categorize genomic functions of the coding sequence, were assigned. For this purpose, the snpEff software was used ${ }^{29}$. The program uses information included in the annotation file (GFF) to assign an annotation to each detected variant. In addition, we predicted effects of nonsynonymous and missense variants on protein functions using the SIFT score. For prediction of effect of amino acid substitution we used SIFT4G software ${ }^{30}$. Variants with SIFT score $\leq 0.05$ were classified as deleterious, while $>0.05$ were considered tolerated ${ }^{31}$.

Statistical analysis. The Shapiro-Wilk test. The Shapiro-Wilk ${ }^{32}$ was used to test whether a sample was obtained from a normal distribution, comparing the following hypotheses:

$H_{0}$ : The distributions of Tajima's $\mathrm{D}$ and nucleotide divergence follow the normal distribution

$H_{1}$ : The distributions of Tajima's D and nucleotide divergence do not follow the normal distribution

The corresponding test statistic is given by:

$$
W=\frac{\left(\sum_{i=1}^{n} a_{i} x_{(i)}\right)^{2}}{\sum_{i=1}^{n}\left(x_{i}-\bar{x}\right)^{2}},
$$

where $a_{i}$ is the tabulated Shapiro-Wilk coefficient, $x_{(i)}$ is the $i$ th smallest value of the Tajima's D/nucleotide divergence and $\bar{x}$ is the mean of Tajima's D/nucleotide divergence. We reject $H_{0}$ at the significance level $(\alpha=0.05)$ if $W<W_{\alpha}$, where $W_{\alpha}$ is tabulated critical threshold for Shapiro-Wilk. 
The $\chi^{2}$ goodness-of-fit test. The $\chi^{2}$ goodness-of-fit test was used to check whether the observed variable in the general population follows the uniform distribution. Corresponding hypotheses were defined as follows ${ }^{33}$ :

$H_{0}$ : The distribution of the observed variable is uniform

$H_{1}$ : The distribution of the observed variable is not uniform

The $\chi^{2}$ goodness-of-fit statistic is given by ${ }^{34}$ :

$$
\chi^{2}=\sum_{i=1}^{k} \frac{\left(O_{i}-E_{i}\right)^{2}}{E_{i}}
$$

where $O_{i}$ is the observed count of observations in the ith group, $E_{i}$ is the count of observations expected under the uniform distribution, and $k$ is the number of groups. Under $H_{0}$, the test follows the $\chi^{2}$ distribution with $k-1$ degrees of freedom.

The Kruskal-Wallis test. The Kruskal-Wallis test is a non-parametric equivalent of the $F$ test in the analysis of variance for non-normal data. Corresponding hypotheses are ${ }^{35}$ :

$H_{0}: \quad$ There is no difference among $k$ populations' median

$H_{1}$ : At least one population's median differs from the median of the other populations

The Kruskal-Wallis statistic is given by ${ }^{36}$ :

$$
H=\frac{12}{N(N+1)} \sum_{i=1}^{k} \frac{R_{i}^{2}}{n_{i}}-3(N+1),
$$

where $N$ is the total sample size, $k$ is the number of groups, $R_{i}$ is the sum of ranks in the $i$ th group, and $n_{i}$ is the size of the $i$ th group. Under $H_{0}$, the distribution of this test can be approximmated by the $\chi^{2}$ distribution with $k-1$ degrees of freedom.

The Mann-Whitney $U$ test. The Mann-Whitney $U$ test is the non-parametric equivalent of the $t$-test for two independent samples with the corresponding hypotheses ${ }^{37}$ :

$H_{0} \quad$ There is no difference between the two populations' medians

$H_{1}$ There is a difference between the two populations' medians

The statistic is given by ${ }^{38,39}$ :

$$
U=M N+\frac{N(N+1)}{2}-\sum_{i=1}^{N} R\left(X_{i}\right),
$$

where $n$ is the size of the first group, $m$ is the size of the second group, and $R\left(X_{i}\right)$ is the rank assigned to the first group (Wilcoxon statistic). We reject the null hypothesis $H_{0}$ at the significance level $\alpha$ if $t_{n, m, 1-\frac{\alpha}{2}}<U<t_{n, m, \frac{\alpha}{2}}$, where $t_{n, m, 1-\frac{\alpha}{2}}$ and $t_{n, m, \frac{\alpha}{2}}$ are quantiles of the Mann-Whitney distribution. For large sample sizes, $U \sim \mathcal{N}\left(\mu_{U}, \sigma_{U}^{2}\right)$, where $\mu_{U}=\frac{m n}{2}, \sigma_{U}^{2}=\frac{m n(N+1)}{12}$, where $N=n+m$. This test was applied to test multiple, simultaneous hypotheses; therefore the Bonferroni correction was used to account for multiple testing ${ }^{40}$.

Genetic statistics. Nucleotide divergence. One of the statistic most widely used to measure the degree of polymorphism in a chromosome is a nucleotide divergence $(\pi)$. It is a measure proposed by Nei and $\mathrm{Li}^{41} . \pi$ quantifies the nucleotide diversity among several sequences. In our case, we estimated $\pi$ along each chromosome in $100 \mathrm{~kb}$ non-overlapping windows. The estimator $\hat{\pi}$ of nucleotide divergence calculated based on information from VCF files is defined as:

$$
\hat{\pi}=\frac{\sum_{i=1}^{k}\left[A N_{i}\left(A N_{i}-1\right)\right]+(w-k) p}{2 \sum_{i=1}^{k}\left[A C_{i}\left(A N_{i}-A C_{i}\right)\right]},
$$

where $k$ is the number of variants within a given $100 \mathrm{~kb}$ window, $A N_{i}$ is the total number of alleles in called genotypes of $i$ th variant, $w$ denotes the window size [ $100 \mathrm{kbs}$ ], $p$ is the number of pairwise windows comparisons, and $A C_{i}$ is the allele count in genotypes of $i$ th variant (for each alternative allele).

Tajima's D. Another statistic used in this study is Tajima's D. It allows for the detection of the evidence of selection. Tajima ${ }^{42}$ proposed this statistic as a measure of a rate of a random (neutral) evolution of DNA sequence. In this study, Tajima’s $D$ was estimated over a $100 \mathrm{kbs}$ non-overlapping windows as follows: 


$$
\hat{D}=\frac{\hat{\theta}_{\pi}-\hat{\theta}_{W}}{\sqrt{\operatorname{Var}\left(\hat{\theta}_{\pi}-\hat{\theta}_{W}\right)}},
$$

where $\hat{\theta}_{\pi}$ is the average number of pairwise differences given by $\hat{\theta}_{\pi}=\frac{\sum_{i<j} d_{i, j}}{\frac{n(n-1)}{2}}$. Here, $d_{i, j}$ represents the number of differences between individual $i$ and $j, n=\left(\begin{array}{c}N \\ 2\end{array}\right)$ is the number of pairwise sequences comparisons with $N$ being the number of individuals. $\hat{\theta}_{W}$ is Watterson's estimator of the expected number of segregating sites under neutrality $\left(\hat{\theta}_{W}=\frac{S}{\sum_{i=1}^{n-1}\left(\frac{1}{i}\right)}\right.$, where $S$ is the number of sites that segregate in the sample).

$D>0$ indicates population reduction or balancing selection, $D=0$ indicates neutral mutations, and $D<0$ indicates population expansion or purifying and positive selection ${ }^{43}$. The VCFtools ${ }^{44}$ was used to calculate the Tajima’s D statistic.

$K_{a} / K_{s}$ ratio. The $K_{a} / K_{s}$ ratio was used as a measure of the selection pressure. Based on this ratio we compared natural selection pressure on proteins between chromosomes ${ }^{45}$. $K_{a}$ represents the ratio of the number of nonsynonymous substitutions per nonsynonymous site, while $K_{s}$ is the number of synonymous substitutions per synonymous site. $K_{a} / K_{s}$ equal to one indicates a neutral selection, the positive ratio is equivalent to positive selection, while the negative ratio indicates purifying selection. The output of the SnpEff ${ }^{29}$ software was used to calculate $K_{a} / K_{s}$ ratio.

Computing environment. All programs and scripts were written in the bash command language and executed on the Genomics High Performance Cluster (GHPC) at the Center for Quantitative Genetics and Genomics at Aarhus University. The computing unit was the Red Hat Enterprise Linux Client release 4.8.5-28 (Centos) with $250 \mathrm{GiB} /$ node of memory and 6 Intel Core Processor CPUs. All statistical analyzes were done using R (version 5.1$)^{46}$ in RStudio ${ }^{47}$ and visualized using the ggplot2 R package ${ }^{48}$.

\section{Data availability}

The subset of the whole-genome data used in this study is available at SRP039339 under PRJNA238491. For the remaining data, the Board of the 1000 Bull Genome Project Consortium should be contacted. Whole-genome sequences from Aarhus University are available only upon agreement with the breeding organization and should be requested directly from the authors.

Received: 30 December 2019; Accepted: 23 July 2020

Published online: 12 August 2020

\section{References}

1. Rosen, B. D. et al. Modernizing the bovine reference genome assembly. Mol. Genet. 3, 802 (2018).

2. Zimin, A. V. et al. A whole-genome assembly of the domestic cow, bos taurus. Genome Biol. 10, R42. https://doi.org/10.1186/gb2009-10-4-r42 (2009).

3. Daetwyler, H. D. et al. Whole-genome sequencing of 234 bulls facilitates mappig of monogenic and complex traits in cattle. Nat. Genet. 46, 858-865 (2014).

4. Chang, T.-C., Yang, Y., Retzel, E. F. \& Liu, W.-S. Male-specific region of the bovine y chromosome is gene rich with a high transcriptomic activity in testis development. Proc. Nat. Acad. Sci. 110, 12373-12378. https://doi.org/10.1073/pnas.1221104110 (2013).

5. Choi, J.-W. et al. Massively parallel sequencing of chikso (Korean brindle cattle) to discover genome-wide SNPs and InDels. Mol. Cells 36, 203-211. https://doi.org/10.1007/s10059-013-2347-0 (2013).

6. VanBuren, R. et al. Extremely low nucleotide diversity in the x-linked region of papaya caused by a strong selective sweep. Genome Biol.https://doi.org/10.1186/s13059-016-1095-9 (2016).

7. Hellborg, L. Low levels of nucleotide diversity in mammalian y chromosomes. Mol. Biol. Evol. 21, 158-163. https://doi.org/10.1093/ molbev/msh008 (2003).

8. Goetting-Minesky, M. P. \& Makova, K. D. Mammalian male mutation bias: impacts of generation time and regional variation in substitution rates. J. Mol. Evol. 63, 537-544. https://doi.org/10.1007/s00239-005-0308-8 (2006).

9. Duret, L. Mutation patterns in the human genome: more variable than expected. PLoS Biol. 7, 1-3. https://doi.org/10.1371/journ al.pbio.1000028 (2009).

10. Amos, W. Even small SNP clusters are non-randomly distributed: is this evidence of mutational non-independence?. Proc. R. Soc. B Biol. Sci. 277, 1443-1449. https://doi.org/10.1098/rspb.2009.1757 (2010).

11. Hodgkinson, A., Ladoukakis, E. \& Eyre-Walker, A. Cryptic variation in the human mutation rate. PLoS Biol. 7, e1000027. https:// doi.org/10.1371/journal.pbio.1000027 (2009).

12. Estivill, X. Chromosomal regions containing high-density and ambiguously mapped putative single nucleotide polymorphisms (SNPs) correlate with segmental duplications in the human genome. Hum. Mol. Genet. 11, 1987-1995. https://doi.org/10.1093/ hmg/11.17.1987 (2002).

13. Varela, M. A. \& Amos, W. Heterogeneous distribution of SNPs in the human genome: microsatellites as predictors of nucleotide diversity and divergence. Genomics 95, 151-159. https://doi.org/10.1016/j.ygeno.2009.12.003 (2010).

14. Aggarwala, V. \& Voight, B. F. An expanded sequence context model broadly explains variability in polymorphism levels across the human genome. Nat. Genet. 48, 349-355. https://doi.org/10.1186/gb-2009-10-4-r421 (2016).

15. Charlesworth, D., Charlesworth, B. \& Marais, G. Steps in the evolution of heteromorphic sex chromosomes. Heredity 95, 118-128. https://doi.org/10.1186/gb-2009-10-4-r422 (2005).

16. Mackiewicz, D., Posacki, P., Burdukiewicz, M. \& Błażej, P. Role of recombination and faithfulness to partner in sex chromosome degeneration. Sci. Rep.https://doi.org/10.1038/s41598-018-27219-1 (2018).

17. Bellott, D. W. et al. Mammalian Y chromosomes retain widely expressed dosage-sensitive regulators. Nature 508, 494-499 (2014).

18. Andrews, S. FastQC A Quality Control tool for High Throughput Sequence Data. http://www.bioinformatics.babraham.ac.uk/ projects/fastqc/ (2014).

19. M Bolger, A., Lohse, M. \& Usadel, B. Trimmomatic: a flexible trimmer for illumina sequence data. Bioinformatics (Oxford, England) 30 (2014). 
20. Li, H. \& Durbin, R. Fast and accurate short read alignment with burrows-wheeler transform. Bioinformatics 25, 1754-1760. https ://doi.org/10.1093/bioinformatics/btp324 (2009).

21. Li, H. et al. The sequence alignment/map format and SAMtools. Bioinformatics 25, 2078-9. https://doi.org/10.1093/bioinforma tics/btp352 (2009).

22. Sims, D., Sudbery, I., Ilott, N. E., Heger, A. \& Ponting, C. P. Sequencing depth and coverage: key considerations in genomic analyses. Nat. Rev. Genet. 15, 121-132. https://doi.org/10.1186/gb-2009-10-4-r424 (2014).

23. Quinlan, A. R. \& Hall, I. M. BEDTools: a flexible suite of utilities for comparing genomic features. Bioinformatics 26, 841-842. https://doi.org/10.1186/gb-2009-10-4-r425 (2010).

24. Picard. https://doi.org/10.1186/gb-2009-10-4-r426.

25. McKenna, A. et al. The genome analysis toolkit: a MapReduce framework for analyzing next-generation DNA sequencing data. Genome Res. 20, 1297-303. https://doi.org/10.1101/gr.107524.110 (2010).

26. de Bruijn, N. A combinatorial problem. Proc. Sect. Sci. Koninklijke Nederlandse Akademie van Wetenschappen te Amsterdam 49, 758-764 (1946).

27. Smith, T. \& Waterman, M. Identification of common molecular subsequences. J. Mol. Biol. 147, 195-197. https://doi.org/10.1186/ $\mathrm{gb}-2009-10-4-\mathrm{r} 428$ (1981).

28. Eilbeck, K. et al. The sequence ontology: a tool for the unification of genome annotations. Genome Biol. 6, R44. https://doi. org/10.1186/gb-2009-10-4-r429 (2005).

29. Cingolani, P. et al. A program for annotating and predicting the effects of single nucleotide polymorphisms, snpeff: Snps in the genome of drosophila melanogaster strain w1118; iso-2; iso-3. Fly 6, 80-92 (2012).

30. Vaser, R., Adusumalli, S., Leng, S. N., Sikic, M. \& Ng, P. C. SIFT missense predictions for genomes. Nat. Protoc. 11, 1-9. https:// doi.org/10.1073/pnas.12211041100 (2015).

31. Ng, P. C. SIFT: predicting amino acid changes that affect protein function. Nucl. Acids Res. 31, 3812-3814. https://doi.org/10.1093/ nar/gkg509 (2003).

32. Shapiro, S. S. \& Wilk, M. B. An analysis of variance test for normality (complete samples). Biometrika 52, 591-611. https://doi. org/10.1073/pnas.12211041102 (1965).

33. Agresti, A. An Introduction to Categorical Data Analysis (Wiley, Hoboken, 2007)

34. Pearson, K. X. on the criterion that a given system of deviations from the probable in the case of a correlated system of variables is such that it can be reasonably supposed to have arisen from random sampling. Lond. Edinb. Dublin Philos. Mag. J. Sci. 50, 157-175. https://doi.org/10.1080/14786440009463897 (1900).

35. Vehkalahti, K. Kruskal-wallis test. In The Concise Encyclopedia of Statistics 288-290 (Springer, New York, 2008). https://doi. org/10.1007/978-0-387-32833-1_216

36. Kruskal, W. H. \& Wallis, W. A. Use of ranks in one-criterion variance analysis. J. Am. Stat. Assoc. 47, 583-621. https://doi. org/10.1080/01621459.1952.10483441 (1952).

37. Vehkalahti, K. Mann-Whitney test. The Concise Encyclopedia of Statistics 327-329 (Springer, New York, 2008). https://doi. org/10.1007/978-0-387-32833-1_243.

38. Wilcoxon, F. Individual comparisons by ranking methods. Biom. Bull. 1, 80. https://doi.org/10.2307/3001968 (1945).

39. Mann, H. B. \& Whitney, D. R. On a test of whether one of two random variables is stochastically larger than the other. Ann. Math. Stat. 18, 50-60. https://doi.org/10.1073/pnas.12211041106 (1947).

40. Dunnett, C. W. A multiple comparison procedure for comparing several treatments with a control. J. Am. Stat. Assoc. 50, $1096-1121$. https://doi.org/10.1080/01621459.1955.10501294 (1955).

41. Nei, M. \& Li, W. H. Mathematical model for studying genetic variation in terms of restriction endonucleases. Proc. Nat. Acad. Sci. 76, 5269-5273. https://doi.org/10.1073/pnas.12211041108 (1979).

42. Tajima, F. Statistical method for testing the neutral mutation hypothesis by DNA polymorphism. Genetics 123, 585-595 (1989).

43. Ezaz, T. \& Edwards, S. V. Editorial: evolutionary feedbacks between population biology and genome architecture. Front. Genet.https ://doi.org/10.3389/fgene.2018.00329 (2018).

44. Danecek, P. et al. The variant call format and VCFtools. Bioinformatics 27, 2156-2158. https://doi.org/10.1007/s10059-013-2347-00 (2011).

45. Hurst, L. D. The ka/ks ratio: diagnosing the form of sequence evolution. Trends Genet. 18, 486-487. https://doi.org/10.1007/s1005 9-013-2347-01 (2002).

46. R Core Team. R: A Language and Environment for Statistical Computing (2018).

47. RStudio Team. RStudio: Integrated Development Environment for R (2016).

48. Wickham, H. ggplot2: Elegant Graphics for Data Analysis (Springer, New York, 2016).

\section{Author contributions}

B.G. and J.S conceived and conducted the experiment, B.C. analyzed the results and wrote the manuscript. All authors reviewed the manuscript.

\section{Competing interests}

The authors declare no competing interests.

\section{Additional information}

Correspondence and requests for materials should be addressed to B.C.

Reprints and permissions information is available at www.nature.com/reprints.

Publisher's note Springer Nature remains neutral with regard to jurisdictional claims in published maps and institutional affiliations.

Open Access This article is licensed under a Creative Commons Attribution 4.0 International License, which permits use, sharing, adaptation, distribution and reproduction in any medium or format, as long as you give appropriate credit to the original author(s) and the source, provide a link to the Creative Commons license, and indicate if changes were made. The images or other third party material in this article are included in the article's Creative Commons license, unless indicated otherwise in a credit line to the material. If material is not included in the article's Creative Commons license and your intended use is not permitted by statutory regulation or exceeds the permitted use, you will need to obtain permission directly from the copyright holder. To view a copy of this license, visit http://creativecommons.org/licenses/by/4.0/.

(c) The Author(s) 2020 